\title{
Neutronic effects of Diagnostic Shield Module length on radiation environment of ITER Diagnostic Generic Upper Port Plug
}

\author{
Arkady Serikov ${ }^{*}$, Luciano Bertalot ${ }^{\mathrm{b}}$, Ulrich Fischera ${ }^{\mathrm{a}}$, Rafael Juarez ${ }^{\mathrm{c}}$
}

${ }^{a}$ Karlsruhe Institute of Technology (KIT), Institute for Neutron Physics and Reactor Technology, Hermann-von-Helmholtz-Platz 1, 76344 Eggenstein-Leopoldshafen, Germany ITER Organization, Route de Vinon-sur-Verdon, CS 90 046, 13067 St. Paul Lez Durance Cedex, France UNED, Calle Juan del Rosal 12, Madrid 28040, Spain

\section{Main objectives of the work}

- Development of the new 3D MCNP neutronics model of the ITER Diagnostic Generic Upper Port Plug (DGUPP) - it is a baseline UP model for insertion into the ITER tokamak standard models (C-lite \& C-Model).

$>$ The model incorporates DGUPP design changes caused by implementation of the ITER Project Change Requests (PCRs) 439 \& 709 concerning the design updates of UPP/blanket interface and UP remote handling.

- Neutronics analysis of DGUPP integrated in UP of the MCNP C-lite Ver. 2 Release R150304 ITER model consisted of $40^{\circ}$ toroidal sector.

$>$ Calculation of radiation 3D maps: neutron flux and Shut-Down Dose Rate (SDDR) in corridors of UPP Inter-Space Structure (ISS), where personnel access is planned $10^{6}$ seconds ( 12 days) of cooling time.

> Minimization of the SDDR in the DGUPP ISS - parametric analysis of the length of the Diagnostic Shielding Module (DSM) by filling with the DSM shielding material all the UP structure till the UP Closure Plate.

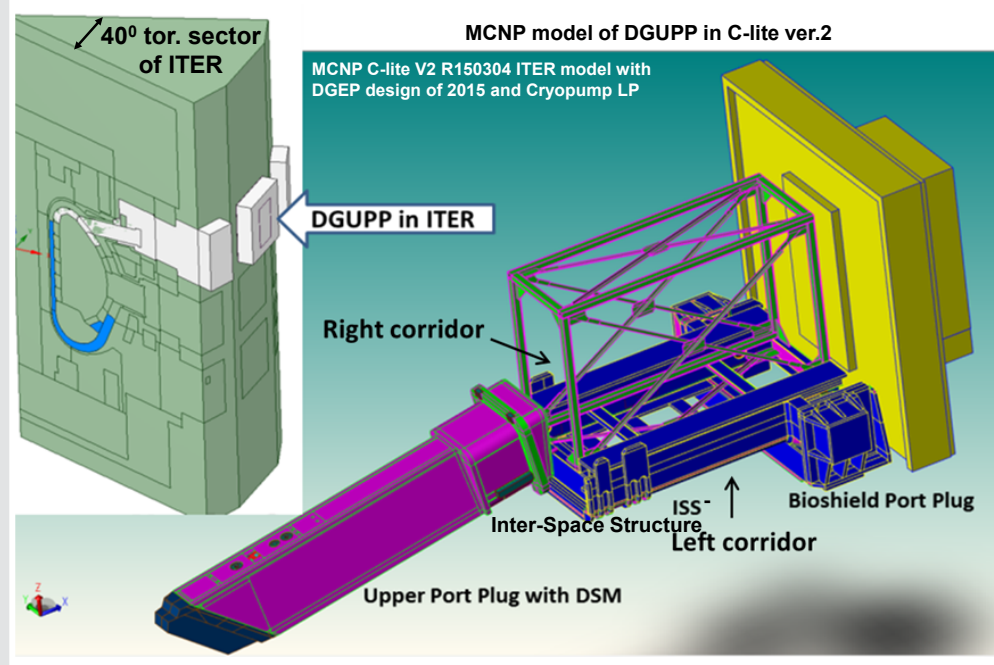

Fusion neutronics computational methodology

\section{State-of-the-art codes approved for ITER \\ - SuperMC (FDS, China) for CAD-to-MCNP conversion: \\ - Activation and SDDR calculations \\ FISPACT-2007 (CCFE) inventory code \\ R2SMesh interface code for MCNP-FISPACT \\ MCNP5 V1.60, MCNP6 (LANL) code;}

The total weight of the Reference DGUPP (IO IDM ref. SZVXHJ_v1_1) The total weight of the Reforence DGUPP (IO IDM ref. SaVXH_vi_1 Volume and material density of the DSM in DGUPP is increased to correspond the 25 ton weight specification.

- In DGUPP the DSM is described as homogenised dummy - DSM material fractions were adjusted (steel, B4C, water, void) to the new drain density of $6.195 \mathrm{~g} / \mathrm{cc}$.

MCNP local model of DGUPP with three

constituent parts of the Diagnostic Shielding Module (DSM) used in following two models (a) and (b): (a) Short-DSM DGUPP with only one DSM part (1)

(b) Long-DSM DGUPP with three DSM parts $(1)+(2)+(3)$.

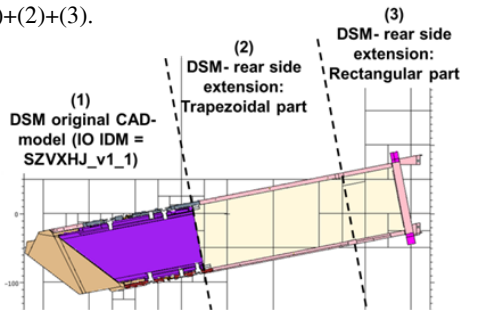

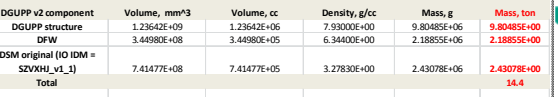

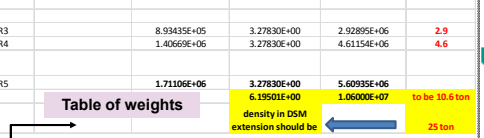

DGUPP with three variants v1-v3: short DSM v1=14.4 ton, den. $3.3 \mathrm{~g} / \mathrm{cm}^{3}$ long DSM $\left(\mathbf{v} 2=20\right.$ ton den. $3.3 \mathrm{~g} / \mathrm{cm}^{3}$ or $\mathbf{v} 3=25$ ton with den. $6.2 \mathrm{~g} / \mathrm{cm}^{3}$ )

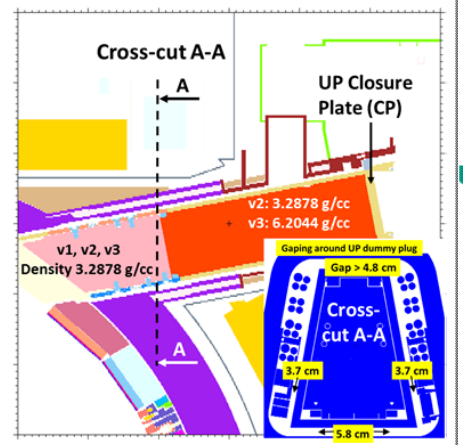

\section{Neutronics effects on DGUPP radiation environment}

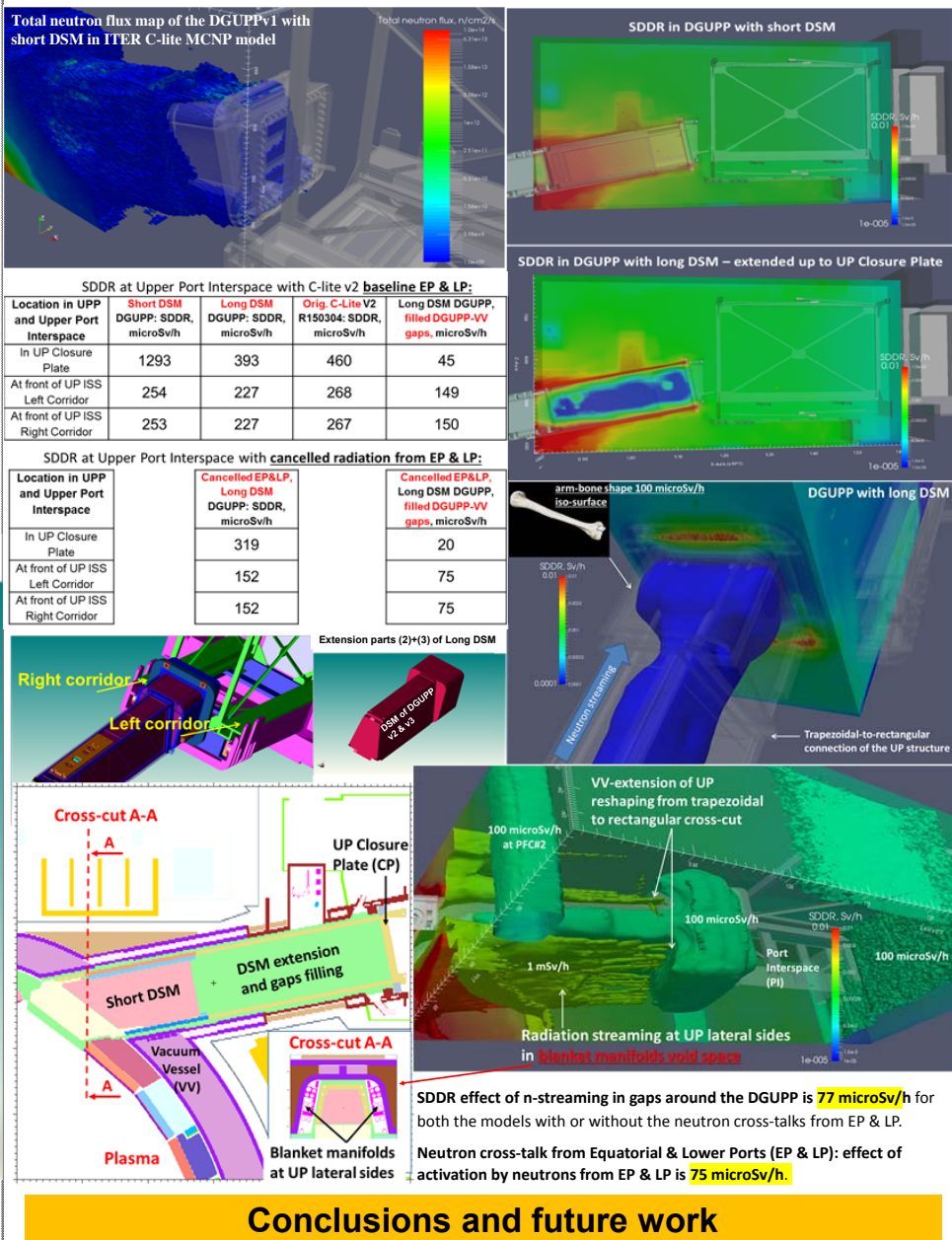

- Design development of the ITER Diagnostic Generic Upper Port Plug (DGUPP) is still in progress. 3D maps of neutron fluxes and Shut-Down Dose Rate (SDDR) with iso-surfaces plotted the DGUPP allowed to find the radiation pathways, hot spots - most critical areas from neutronics perspectives.

Revealed radiation streaming along the bottom and side gaps and inside the empty space of port structure behind the short Diagnostic Shielding Module (DSM) motivated the need to improve further the design of DSM. In all cases, the shielding design improvement should follow the ALARA principle, using the low activated materials with reduced contents of impurities - parent isotopes contributed to short and long term SDDR ( $\mathrm{Co}, \mathrm{Ta}, \mathrm{Ni}, \mathrm{Nb})$.

- A variant of Long DSM has been analyzed. The engineering implementation of the Long DSM option is still under consideration. Along that, particular attention should be devoted to shielding insertion at the trapezoidal-to-rectangular reshaping of the UP rear structure. At this place neutron streaming could be stopped most effectively. Presented neutronics results were obtained in parametric study of the DGUPP shielding performance. These results are not absolute, they depend on other systems of ITER model C-lite V2 of 2015, which was updated afterwards to .

- A study has been carried out Neutronic investigation is planning for further DGUPP improvement and SDDR reduction by taking into account the updated ITER C-Model and by aiming to find the realistic engineering design solutions.

\section{Acknowledgment \& Disclaimer}

This work has been funded by the ITER Organization under the ITER contract Nr. 1O/4300000896.

The views and opinions expressed herein do not necessarily reflect those of the ITER Organization. 